\title{
TRIM9 Mediates Netrin-1-Induced Neuronal Morphogenesis in the Developing and Adult Hippocampus
}

\author{
ㄴovane Aiken ${ }^{1}$ and ${ }^{\circledR G}$ Georgia Buscaglia ${ }^{2}$ \\ ${ }^{1}$ Cell Biology, Stem Cells, and Development Program and ${ }^{2}$ Neuroscience Program, University of Colorado School of Medicine, Aurora, Colorado 80045 \\ Review of Winkle, Olsen et al.
}

Proper formation of the hippocampus and continued integration of adult-born hippocampal neurons into hippocampal circuitry are critical for learning and memory. During embryonic development, hippocampal neurons undergo dramatic morphological changes to migrate to the correct layer of the hippocampus and extend axons and dendrites to synaptic targets. Neural stem and progenitor cells in the subgranular zone of the hippocampal dentate gyrus continue to generate excitatory principal neurons, or granule cells, throughout the life of all mammals investigated to date, including humans (Eriksson et al., 1998). As adult-born granule cells are generated, they recapitulate many of the morphological stages that occur during embryonic development to be appropriately incorporated into the existing neuronal circuitry. The generation and functional integration of hippocampal neurons appear to depend on a number of factors, including intrinsic and extrinsic regulatory factors, environmental stimuli, and appropriate synaptic input (Ehninger and Kempermann, 2008). Although

Received June 14, 2016; revised Aug. 1, 2016; accepted Aug. 2, 2016.

J.A. was supported by the National Science Foundation Graduate Research Fellowships Program grant number 1000203326. G.B. was supported by the Neuroscience Training Grant T32 HD 041697. We thank Marina Picciotto and Teresa Esch for critical review of the manuscript; and members of the Developmental Neurobiology course for their input, especially course directors Emily Bates and Steven Britt.

The authors declare no competing financial interests.

Correspondence should be addressed to Jayne Aiken, University of Colorado School of Medicine, 12801 East 17th Place, MS8108, Aurora, C0 80045. E-mail: jayne.aiken@ucdenver.edu.

DOI:10.1523/JNEUROSCI.1917-16.2016

Copyright $\odot 2016$ the authors $\quad 0270-6474 / 16 / 369513-03 \$ 15.00 / 0$ many studies have elucidated several molecular factors regulating adult neurogenesis, the molecular factors that regulate morphogenesis and integration have not been well characterized.

One protein that might regulate hippocampal development is tripartite motif containing 9 (TRIM9), a member of the tripartite motif family of E3 ubiquitin ligases. TRIM9 is highly expressed in the cerebral cortex and hippocampus (Tanji et al., 2010), and TRIM9 expression is reduced in the brains of patients with memory loss neuropathologies, including dementia with Lewy bodies (Tanji et al., 2010). Moreover, recent studies have demonstrated an important role for TRIM9 in regulating neuronal branching and axon guidance (Winkle et al., 2014; Menon et al., 2015). Specifically, TRIM9 modulates morphological changes induced by the secreted guidance molecule, Netrin-1, in cortical neurons (Winkle et al., 2014).

Netrin-1 signaling promotes neuronal arborization and synaptogenesis in numerous neuronal systems, including cortical and hippocampal neurons (Barallobre et al., 2000; Winkle et al., 2014). TRIM9 interacts directly with multiple factors, such as vasodilator-stimulated phosphoprotein (VASP) and synaptosome-associated protein $25 \mathrm{kDa}$ (SNAP25), to help mediate the neuronal response to Netrin-1 through ligase-dependent and -independent actions (Fig. 1). In the absence of Netrin-1, TRIM9 interacts directly with SNAP25 (Winkle et al., 2014), a member of the SNARE complex important for exocytosis, and VASP
(Menon et al., 2015), a filamentous actin polymerization factor (Fig. 1). Interactions of TRIM9 with SNAP25 and VASP lead to inhibition of membrane insertion and actin polymerization, respectively, both of which suppress neurite growth and branching. Although TRIM9 ligase activity is essential for VASP ubiquitination, TRIM9 acts on SNAP25 in a ligase-independent manner. Upon Netrin-1 introduction, TRIM9 binds the Netrin-1 receptor deleted in colorectal cancer (DCC), and TRIM9's other known interactions are inhibited, thus promoting membrane expansion, filopodia advancement, and axon guidance.

Another intriguing role of TRIM9 in $\mathrm{Ne}$ trin-1 signaling is its potential control of DCC receptor localization to the membrane. Upon Netrin-1 stimulation, TRIM9 inhibition of exocytosis is relieved and VAMP2- and VAMP7-positive vesicles can fuse with the plasma membrane (Winkle et al., 2014). Gopal et al. (2016) recently revealed that Netrin-1/DCC activation leads to DCC recruitment to the plasma membrane through exocytosis. DCC is associated with both VAMP2- and VAMP7-containing vesicles (Cotrufo et al., 2012). As a result of this membrane expansion, more DCC is added to the membrane, thus possibly amplifying Netrin-1 signaling. This provides a novel means by which Netrin-1-induced TRIM9 inhibition may propagate the $\mathrm{Ne}$ trin-1 signal.

A recent report in The Journal of Neuroscience provides compelling evidence to support the hypothesis that TRIM9 is critical for regulating morphogenesis of hip- 
pocampal neurons, and ultimately aiding learning and memory (Winkle, Olsen et al., 2016). Winkle, Olsen et al. (2016) first confirm reports of high levels of TRIM9 protein (Tanji et al., 2010) in the hippocampus and demonstrate that TRIM9 colocalizes and coimmunoprecipitates with DCC. The interaction between TRIM9 and DCC in hippocampal neurons suggests that TRIM9 may help mediate Netrin-1 effects as it does in cortical neurons (Winkle et al., 2014).

In primary hippocampal cultures lacking Trim9, neurons initiated neurite outgrowth prematurely, maintained more neurites throughout maturation, and ultimately exhibited more complex dendritic arborization than Trim $9^{+/+}$control cells. These data suggest that TRIM9 normally inhibits neurite formation and arborization during neuron morphogenesis in the hippocampus, supporting previous findings.

Upon Netrin-1 treatment, Trim $9^{+/+}$ cells exhibited accelerated neurite growth, whereas Trim $9^{-1-}$ cells did not. TRIM9 ligase activity and DCC binding were required for Netrin-1 sensitivity, but not for TRIM9-mediated restraint of neurite growth. This is surprising given that TRIM9 was previously thought to restrict neurite outgrowth, in part by inhibiting filopodia formation through ligase-dependent VASP ubiquitination. However, this result suggests that either the ligase-independent TRIM9 interaction with SNAP25 is sufficient to inhibit neurite outgrowth or that TRIM9 may act beyond its known interactions to inhibit neurite outgrowth (Fig. 1) (Menon et al., 2015). These data suggest that loss of TRIM9 mimics the effect of endogenous Netrin-1 activity by releasing inhibition of neurite outgrowth. However, additional information is required to understand how TRIM9 ubiquitin ligase activity propagates the Netrin-1 signal.

In addition to investigating TRIM9's role in dendritic arborization, Winkle, Olsen et al. (2016) examined how TRIM9 affects axon pathfinding. Because Netrin-1 signaling is required for establishing axonal projections between CA3 pyramidal cells and the septal nuclei (Barallobre et al., 2000), the authors investigated how conditional deletion of TRIM9 in excitatory cortical and hippocampal neurons affected CA3-to-septal projections in vivo. Because TRIM9 mediates Netrin-1 signaling in vitro and in cortical neurons (Winkle et al., 2014; Menon et al., 2015), the authors hypothesized that loss of TRIM9 would reduce the density of CA3-to-septal nuclei projections, as was observed after Netrin-1 deletion (Barallobre et al., 2000). In contrast to this hypothesis, the authors noted an increased

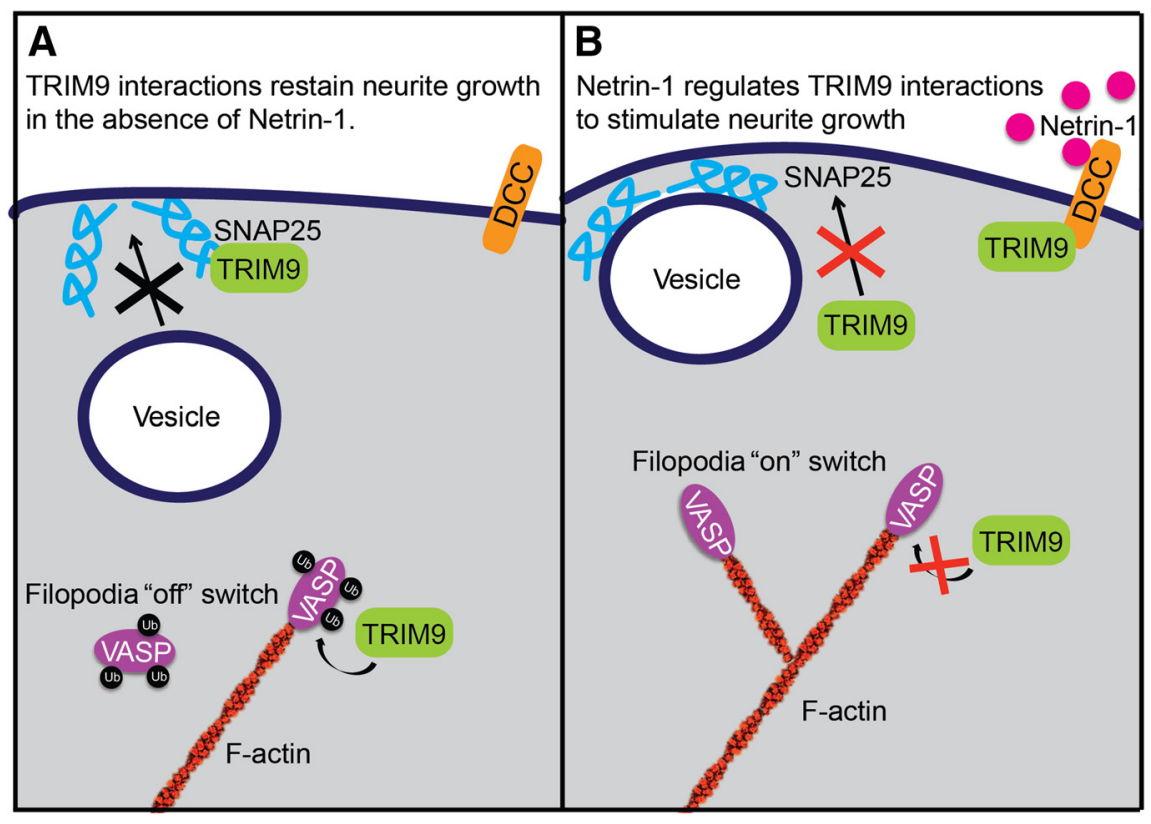

Figure 1. Known TRIM9 interactions. $\boldsymbol{A}$, In the absence of Netrin-1, TRIM9 inhibits SNAP25 in a ligase-independent manner to inhibit exocytosis. TRIM9 also acts as a filopodia "off" switch by ubiquitinating actin polymerase VASP. Ubiquitinated VASP is dissociated from the ends of actin filaments, leading to decreased actin polymerization. $\boldsymbol{B}$, When Netrin-1 is present, TRIM9 interacts with Netrin-1 receptor DCC in a ligase-dependent manner. TRIM9's interaction with SNAP25 is inhibited upon Netrin-1 introduction, leading to exocytosis-mediated membrane expansion. TRIM9 ubiquitination of VASP is also inhibited, leading to VASP association with actin filaments, increased actin filament polymerization, and promotion of filopodia formation. F-actin, filamentous actin; Ub, ubiquitination.

density of fibers in the septal nuclei of Trim $9^{-1-}$ mice. The authors speculated that this was due to an increase of aberrant cortical projections invading this region. However, as these experiments were performed using the Nex-Cre promoter, which drives TRIM9 loss in the hippocampus but not in the septum (Goebbels et al., 2006), an additional possibility is that the presence of TRIM9 in the postsynaptic neurons of the septum is sufficient to promote Netrin-1mediated axon outgrowth. Although the postsynaptic role of TRIM9 has not been investigated, TRIM9 was found to be enriched in the postsynaptic density (Jordan et al., 2004). Future studies are needed to determine the role of TRIM9 in the postsynaptic density. Further, as Netrin-1 is known to have interactions with a multitude of other proteins, it is unsurprising that the distinct aberrant hippocampal projections observed in Netrin-1 deletion cannot be attributed to interactions with TRIM9 alone.

Importantly, Winkle, Olsen et al. (2016) also investigated the role of TRIM9 in adultborn hippocampal neurons in the dentate gyrus. As expected, Trim $9^{-1-}$ adult-born granule cells exhibited dendritic hyperarborization. Notably, Trim $9^{-/-}$adult-born granule cells incorrectly migrated past the inner third region of the granule cell layer to inappropriate areas, such as the hilus, where Netrin-1 is normally not expressed. How- ever, Netrin-1 can be induced in this region by epileptic activity, which similarly leads to aberrant neuron migration to the hilus (Yang et al., 2008). Together, these data suggest that loss of TRIM9 replicates the effect of aberrant Netrin-1 expression. These intriguing results provide evidence that Netrin-1 cues are important for guiding adult-born hippocampal neuron migration and that perhaps TRIM9 normally helps mediate a Netrin-1 signal found in the adult hippocampus.

To investigate the behavioral outcome of Trim 9 loss in mice, the authors used behavioral assays to measure motor function, anxiety, social interaction, exploratory behavior, and learning and memory. Amazingly, the authors report that Trim $9^{-1-}$ mice exhibited few behavioral defects, with the exception of learning and memory deficits, as tested by the Morris water maze. Interestingly, the Morris water maze is a stressful behavioral test for mice (Garthe and Kempermann, 2013), and Trim9 $9^{-1-}$ mice also exhibited moderate deficits in other anxiety-related behavioral tasks, such as the open field test. Although anxiety was dismissed as an unlikely cause of these deficits due to a lack of difference between groups in the elevated plus maze, it would be useful to examine how these mice perform on a nonstressful hippocampal task, such as the T-maze spontaneous alternation task 
(Gerlai, 2001). The highly specific behavioral deficits are also surprising in light of reports that Trim9 loss causes neuronal arborization and axon guidance defects in cortical neurons (Winkle et al., 2014; Menon et al., 2015). Further studies may be required to examine higher cognitive function in Trim $9^{-1-}$ mice.

Although Winkle, Olsen et al. (2016) provide compelling evidence for TRIM9 influencing hippocampal neuron development, numerous questions remain regarding its mechanism of action. Recent studies have focused on Netrin-1-mediated TRIM9 activity and have revealed many ways in which TRIM9 activity is altered upon Netrin-1/DCC activation (Fig. 1). This report by Winkle, Olsen et al. (2016) is consistent with these TRIM9 roles but does not explain how TRIM9 modulates the Netrin-1/DCC signal. Upon Netrin-1/DCC binding, DCC P3 domains homodimerize (Xu et al., 2014) and activate poorly understood downstream signaling mechanisms to ultimately drive cytoskeletal reorganization (Lai Wing Sun et al., 2011). This report and others reveal that TRIM9 directly binds DCC and that its ligase activity is important for $\mathrm{Ne}$ trin-1 signal propagation (Winkle et al., 2014; Menon et al., 2015; Winkle, Olsen et al., 2016). However, whether TRIM9 acts on DCC to modulate its behavior or acts on other substrates in the Netrin-1 pathway remains unanswered. A comprehensive understanding of TRIM9's opposing mechanisms during Netrin-1 signaling requires further study to explore Netrin-1-induced changes to TRIM9 activity, as well as to identify and investigate other TRIM9 substrates and binding partners.

\section{References}

Barallobre MJ, Del Río JA, Alcántara S, Borrell V, Aguado F, Ruiz M, Carmona MA, Martín M, Fabre M, Yuste R, Tessier-Lavigne M, Soriano E (2000) Aberrant development of hippocampal circuits and altered neural activity in netrin 1-deficient mice. Development 127: 4797-4810. Medline

Cotrufo T, Andrés RM, Ros O, Pérez-Brangulí F, Muhaisen A, Fuschini G, Martínez R, Pascual M, Comella JX, Soriano E (2012) Syntaxin 1 is required for DCC/Netrin-1-dependent chemoattraction of migrating neurons from the lower rhombic lip. Eur J Neurosci 36:31523164. CrossRef Medline

Ehninger D, Kempermann G (2008) Neurogenesis in the adult hippocampus. Cell Tissue Res 331:243-250. CrossRef Medline

Eriksson PS, Perfilieva E, Björk-Eriksson T, Alborn AM, Nordborg C, Peterson DA, Gage FH (1998) Neurogenesis in the adult human hippocampus. Nat Med 4:1313-1317. CrossRef Medline

Garthe A, Kempermann G (2013) An old test for new neurons: refining the Morris Water Maze to study the functional relevance of adult hippocampal neurogenesis. Front Neurosci 7: 1-11. CrossRef Medline

Gerlai R (2001) Behavioral tests of hippocampal function: simple paradigms complex problems. Behav Brain Res 125:269-277. CrossRef Medline

Goebbels S, Bormuth I, Bode U, Hermanson O, Schwab MH, Nave KA (2006) Genetic targeting of principal neurons in neocortex and hippocampus of NEX-Cre mice. Genesis 44: 611-621. CrossRef Medline

Gopal AA, Rappaz B, Rouger V, Martyn IB, Dahlberg PD, Meland RJ, Beamish IV, Kennedy TE, Wiseman PW (2016) Netrin-1-Regulated Distribution of UNC5B and DCC in Live Cells Re- vealed by TICCS. Biophys J 110:623-634. CrossRef Medline

Jordan BA, Fernholz BD, Boussac M, Xu C, Grigorean G, Ziff EB, Neubert TA (2004) Identification and verification of novel rodent postsynaptic density proteins. Mol Cell Proteomics 3:857-871. CrossRef Medline

Lai Wing Sun K, Correia JP, Kennedy TE (2011) Netrins: versatile extracellular cues with diverse functions. Development 138: 2153-2169. CrossRef Medline

Menon S, Boyer NP, Winkle CC, McClain LM, Hanlin CC, Pandey D, Rothenfußer S, Taylor AM, Gupton SL (2015) The E3 ubiquitin ligase TRIM9 is a filopodia off switch required for Netrin-dependent axon guidance. Dev Cell 35:698-712. CrossRef Medline

Tanji K, Kamitani T, Mori F, Kakita A, Takahashi H, Wakabayashi K (2010) TRIM9, a novel brain-specific E3 ubiquitin ligase, is repressed in the brain of Parkinson's disease and dementia with Lewy bodies. Neurobiol Dis 38 : 210-218. CrossRef Medline

Winkle CC, McClain LM, Valtschanoff JG, Park CS, Maglione C, Gupton SL (2014) A novel Netrin-1-sensitive mechanism promotes local SNARE-mediated exocytosis during axon branching. J Cell Biol 205:217-232. CrossRef Medline

Winkle CC, Olsen RH, Kim H, Moy SS, Song J, Gupton SL (2016) Trim9 deletion alters the morphogenesis of developing and adult-born hippocampal neurons and impairs spatial learning and memory. J Neurosci 36:49404958. CrossRef Medline

Xu K, Wu Z, Renier N, Antipenko A, Tzvetkova-Robev D, Xu Y, Minchenko M, Nardi-Dei V, Rajashankar KR, Himanen J, Tessier-Lavigne M, Nikolov DB (2014) Neural migration: structures of netrin-1 bound to two receptors provide insight into its axon guidance mechanism. Science 344:12751279. CrossRef Medline

Yang F, Wang JC, Han JL, Zhao G, Jiang W (2008) Different effects of mild and severe seizures on hippocampal neurogenesis in adult rats. Hippocampus 18:460-468. CrossRef Medline 